\title{
A closer look on neural inertia during anesthesia with different drug combinations in a controlled step-up/step-down design in humans
}

\author{
Kuizenga M.H.1
}

1. University Medical Center Groningen, University of Groningen, Groningen, The Netherlands.

2. Ghent University, Ghent, Belgium.

university of

groningen

3. AZ Sint-Jan Brugge-Oostende AV, Brugge, Belgium.

\section{Background}

- Neural inertia (NI) is defined as the tendency of the central nervous system to resist transitions between arousal states, potentially observable as a hysteresis in clinical signs and during neurophysiologic monitoring between induction and recovery

- This phenomenon has been observed in mice

and drosophila with volatile anesthetics by demonstrating a higher required anesthetic concentration during induction than during recovery to switch between states (induction $\mathrm{C}_{50}>$ recovery $\left.\mathrm{C}_{50}\right)(1)$

\section{Goal of Study}

To evaluate this phenomenon in humans using propofol or sevoflurane (both with or without remifentanil) as anesthetic agents.

\section{Materials \& Methods}

- 36 healthy volunteers received four sessions of anesthesia with different drug combinations in a step-up/step-down design

- During these sessions propofol or sevoflurane was administered with or without remifentanil $\left(0,2\right.$ or $\left.4 \mathrm{ng} \mathrm{mL}^{-1}\right)$

- Serum concentrations of propofol and remifentanil were measured from arterial blood samples in steady state conditions

- Loss and return of responsiveness (LOR-ROR), response to pain (PAIN), Patient State Index (PSI) and 95\% spectral edge frequency (SEF) were recorded and modeled with NONMEM to fit a sigmoidal $\mathrm{E}_{\max }$ dose response relationship incorporating the fit of neural inertia

\begin{tabular}{|c|c|c|c|}
\hline$C_{\text {REMI }}$ & $\begin{array}{c}\mathbf{0} \mathbf{n g ~ m L}^{-1} \\
\text { (Group P \& S) }\end{array}$ & $\begin{array}{c}\mathbf{2} \mathbf{~ n g ~ m L}^{-1} \\
\text { (50\% of Group PR \& SR) }\end{array}$ & $\begin{array}{c}\mathbf{4} \mathbf{n g ~ m L}^{-1} \\
\text { (50\% of Group PR \& SR) }\end{array}$ \\
\hline Age (Years) & Males/Females & Males/Females & Males/Females \\
\hline $\mathbf{1 8 - 3 5}$ & $6 / 6$ & $3 / 3$ & $3 / 3$ \\
\hline $\mathbf{3 5 - 5 0}$ & $6 / 6$ & $3 / 3$ & $3 / 3$ \\
\hline $\mathbf{5 0 - 7 0}$ & $6 / 6$ & $3 / 3$ & $3 / 3$ \\
\hline $\begin{array}{c}\text { Total number } \\
\text { Males/Females }\end{array}$ & $18 / 18$ & $9 / 9$ & $9 / 9$ \\
\hline
\end{tabular}

Table 1. Stratification of 36 volunteers according to age, gender and remifentanil effect-site concentration $\left(\mathrm{Ce}_{\mathrm{REMI}}\right)$.

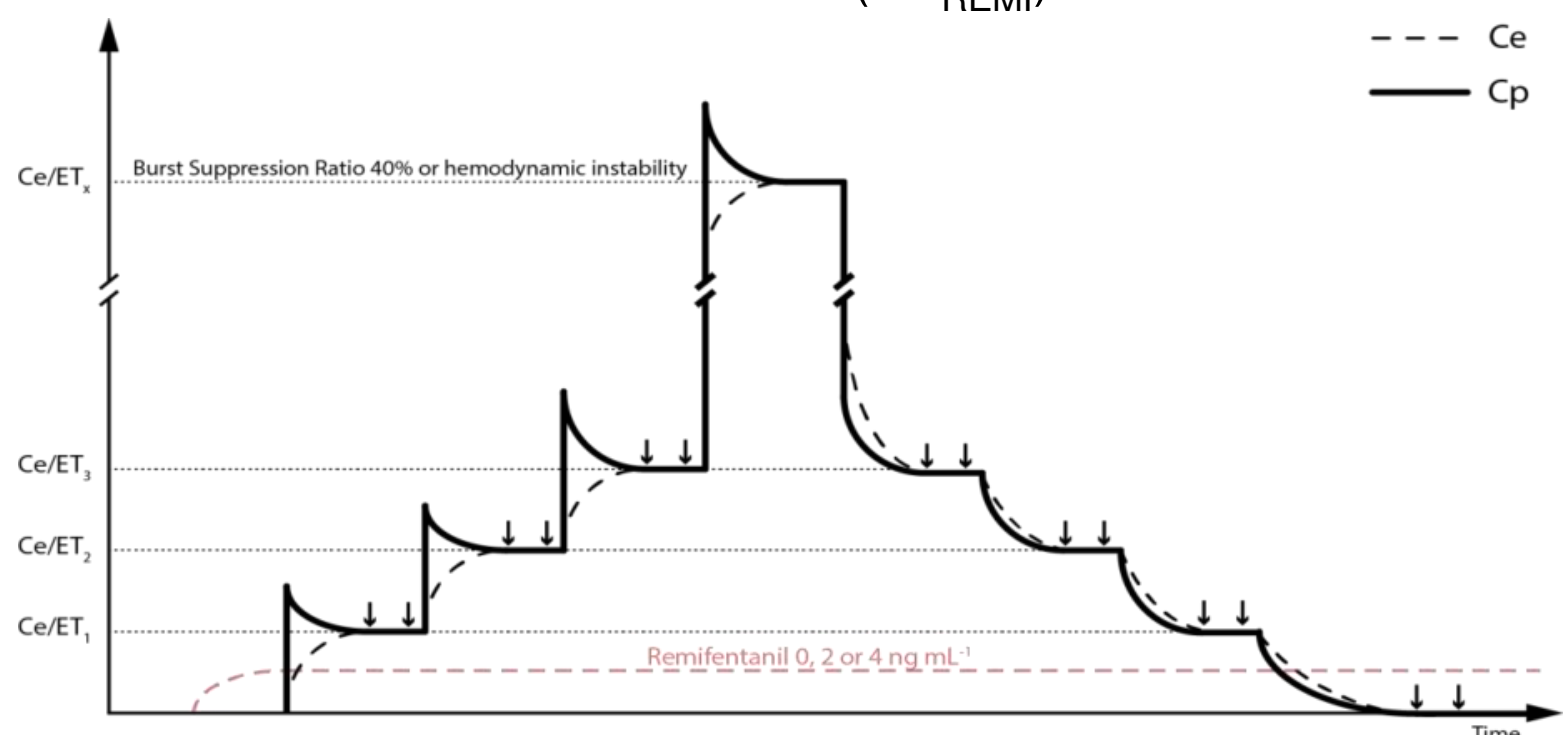

Figure 1. 'Staircase' step-up and step-down administration.

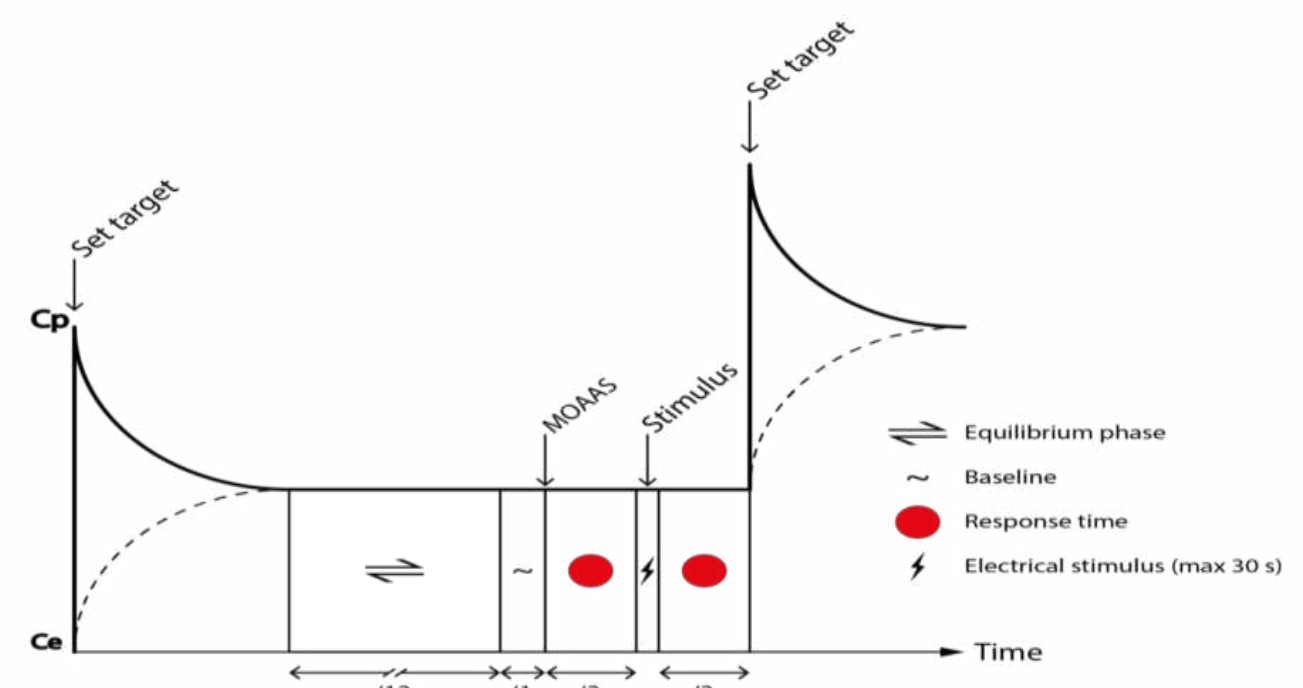

Figure 2. Observations of responsiveness in pseudo-steady state.

\begin{tabular}{|c|c|c|c|c|c|c|c|c|c|}
\hline & & $\mathrm{Re}$ & Jlts & 2 & SC & is & & & \\
\hline & & LOF & ROR & & & & & & \\
\hline & & Prop & Sevo & Prop & Sevo & Prop & Sevo & Prop & Sevo \\
\hline Baseline & $E_{0}$ & - & - & - & - & 84.6 & & 21.2 & 2.3) \\
\hline & $E_{\max }$ & - & - & - & - & -65. & (1.9) & $\begin{array}{c}-12.4 \\
(4.9)\end{array}$ & $\begin{array}{r}-11.3 \\
(5.5)\end{array}$ \\
\hline $\begin{array}{l}\text { Drug } \\
\text { effoct }\end{array}$ & $\mathrm{C}_{50}$ & $\begin{array}{l}1.72 \\
(5.4)\end{array}$ & $\begin{array}{c}0.662 \\
(6.0)\end{array}$ & $\begin{array}{c}2.78 \\
(12.7)\end{array}$ & $\begin{array}{c}0.825 \\
(9.3)\end{array}$ & $\begin{array}{l}1.76 \\
(5.1)\end{array}$ & $\begin{array}{l}1.02 \\
(4.6)\end{array}$ & $\begin{array}{l}2.36 \\
(5.7)\end{array}$ & $\begin{array}{l}1.44 \\
(6.8)\end{array}$ \\
\hline & Y & $\begin{array}{c}5.17 \\
(12.8)\end{array}$ & $\begin{array}{c}6.55 \\
(12.2)\end{array}$ & $\begin{array}{c}2.53 \\
(10.9)\end{array}$ & $\begin{array}{l}3.34 \\
(9.8)\end{array}$ & 2.97 & (5.2) & 3.59 & 12.8) \\
\hline & Slope & - & & & 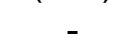 & - & - & - & - \\
\hline Remi & $\theta_{1}$ & $\begin{array}{c}-0.28 \\
(15.4)\end{array}$ & $\begin{array}{l}-0.23 \\
(27.7)\end{array}$ & $\begin{array}{c}-0.669 \\
(7.7)\end{array}$ & $\begin{array}{c}-0.543 \\
(11.5)\end{array}$ & $\begin{array}{c}-0.22 \\
(14.7)\end{array}$ & $\begin{array}{l}-0.17 \\
(30.0)\end{array}$ & $\begin{array}{c}-0.37 \\
(14.3)\end{array}$ & $\begin{array}{l}-0.11 \\
(62.5)\end{array}$ \\
\hline action & $\theta_{2}$ & $\begin{array}{c}0.77 \\
(40.4)\end{array}$ & $\begin{array}{c}0.88 \\
(59.8)\end{array}$ & $\begin{array}{c}0.24 \\
(38.6)\end{array}$ & $\begin{array}{l}0.31 \\
(54)\end{array}$ & NS & NS & NS & NS \\
\hline & $\theta_{3}$ & NS & NS & NS & NS & NS & $\begin{array}{l}0.125 \\
(34.1)\end{array}$ & NS & NS \\
\hline $\begin{array}{l}\text { Neural } \\
\text { inertia }\end{array}$ & $\theta_{4}$ & NS & $\begin{array}{l}0.184 \\
(40.3)\end{array}$ & NS & NS & NS & $\begin{array}{l}0.343 \\
(29.1)\end{array}$ & NS & NS \\
\hline & $\theta_{5}$ & NS & $\begin{array}{l}0.402 \\
(41.3)\end{array}$ & NS & $\begin{array}{l}0.599 \\
(43.6)\end{array}$ & NS & NS & NS & NS \\
\hline & $\mathrm{E}_{0}{ }^{1}$ & - & & & & & & 8.38 & \\
\hline IIV & $\mathrm{C}_{50}{ }^{1}$ & $\begin{array}{l}2.6 \\
(37.2)\end{array}$ & $\begin{array}{r}18.5 \\
(42.1)\end{array}$ & $\begin{array}{l}5.9 \\
(30.9)\end{array}$ & $\begin{array}{l}27.1 \\
(37.8)\end{array}$ & $\begin{array}{c}24.3 \\
(24.0)\end{array}$ & $\begin{array}{c}20.9 \\
(33.9)\end{array}$ & $\begin{array}{c}36.2 \\
(29.6)\end{array}$ & $\begin{array}{c}33.9 \\
(55.1)\end{array}$ \\
\hline & $\rho C_{50}$ & & & & 43.3) & & 33.5) & 0.85 & 45.2) \\
\hline UV & $\sigma_{\text {Ad }}$ & & & & & 9.5 & 11.1) & 3.5 & $9.2)$ \\
\hline
\end{tabular}

Table 2. The model parameters $\left(E_{0}, E_{\max }, C_{50}, Y\right)$ for the various pharmacodynamic endpoints (LOR-ROR, PAIN, PSI, SEF) related to the measured concentration of propofol or sevoflurane in pseudosteady state condition (C), the influence of remifentanil $2 \mathrm{ng} \mathrm{mL}^{-1}\left(\theta_{1}\right)$ and $4 \mathrm{ng} \mathrm{mL}^{-1}\left(\theta_{2}\right)$, and possible neural inertia on the model. More specifically, $\theta_{3}, \theta_{4}$ and $\theta_{5}$ estimate the increase in $\mathrm{C}_{50}$ for the induction phase as compared to the recovery for the 0,2 or $4 \mathrm{ng} \mathrm{mL}^{-1}$ remifentanil groups, respectively.

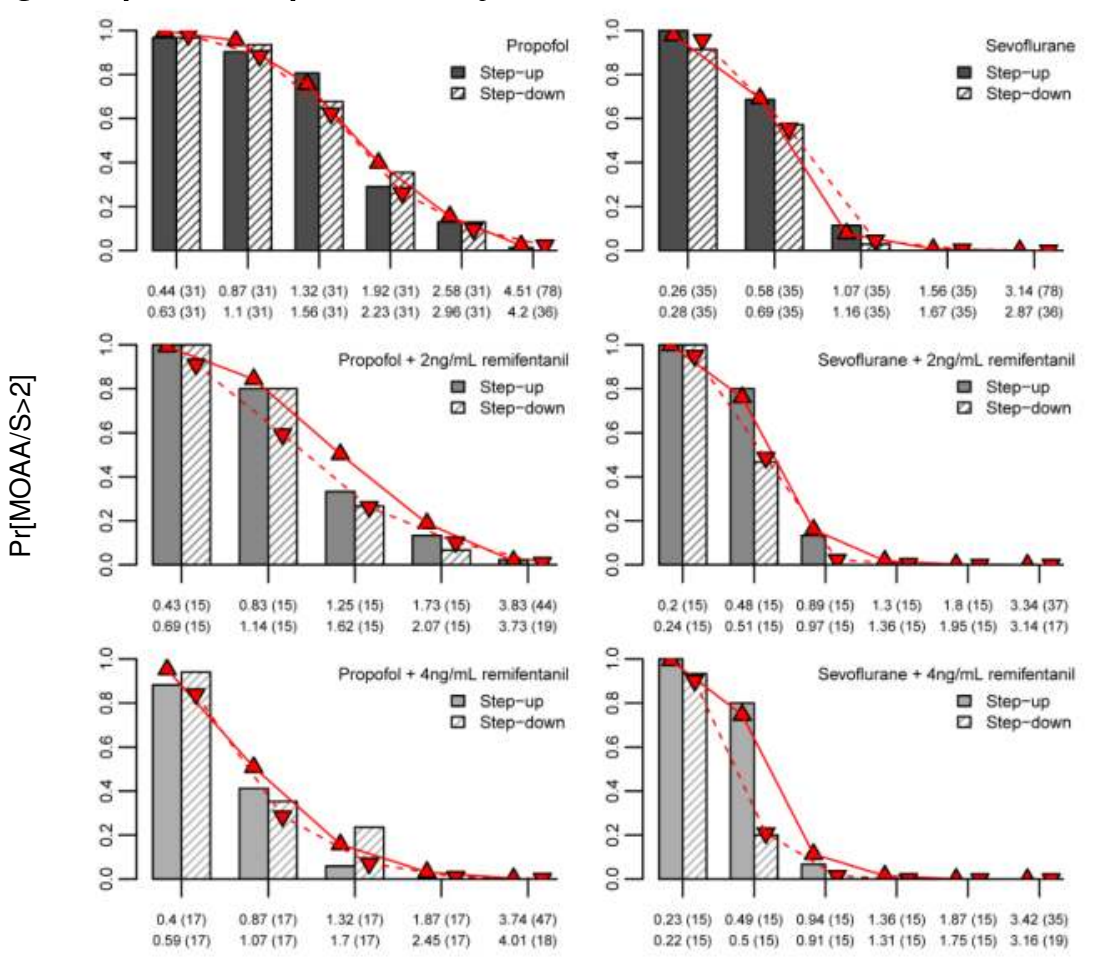

Figure 3. Barplots of the observed and predicted responses to a verbal command (LOR-ROR).

\section{Conclusions}

- Our results nuance the earlier findings with volatile anesthetics in mice and drosophila

- Methodological aspects of the study, such as the measured endpoint, have an effect on the detection of $\mathrm{NI}$

- A more thorough definition of $\mathrm{NI}$, accompanied by a robust methodological framework for clinical studies is required to advance our knowledge of this phenomenon

\section{References}

(1) Friedman EB, Sun $Y$, Moore JT, Hung $H$, Meng QC, Perera $P$, et al. A conserved behavioral state barrier impedes transitions between anesthetic-induced unconsciousness and wakefulness: evidence for neural inertia. PLoS ONE 2010;5(7):e11903 